\title{
AN ELEMENTARY PROOF OF JAMES' CHARACTERIZATION OF WEAK COMPACTNESS
}

\author{
WARREN B. MOORS
}

(Received 1 November 2010)

\begin{abstract}
In this paper we provide an elementary proof of James' characterization of weak compactness in separable Banach spaces. The proof of the theorem does not rely upon either Simons' inequality or any integral representation theorems. In fact the proof only requires the Krein-Milman theorem, Milman's theorem and the Bishop-Phelps theorem.
\end{abstract}

2010 Mathematics subject classification: primary 46B20; secondary 46B22.

Keywords and phrases: compact convex sets, boundaries, Rainwater's theorem, Krein-Milman theorem.

Ever since James first proved in [6] that, in any Banach space $X$, a closed bounded convex subset $C$ of $X$ is weakly compact if, and only if, every continuous linear functional attains its supremum over $C$, there has been continued interest in trying to simplify his proof. Some success was achieved in [5] when Godefroy used Simons' inequality [14] to deduce James' theorem in the case of a separable Banach space. However, although the proof of Simons' inequality is elementary, it is certainly not easy and so the search for a simple proof continued. Later in [4] Fonf et al. used the notion of $(I)$-generation to provide an alternative proof of James' theorem (in the separable Banach space case) without recourse to Simons' inequality (this was later slightly refined in [3]). Their proof was short and reasonably elementary. However, it still relied upon integral representation theorems, as well as the Bishop-Phelps theorem [2]. In this short note we show how to modify the proof in [4] in order to further simplify matters by replacing the integral representations in [4] by the use of the Krein-Milman theorem [8] in conjunction with Milman's theorem [9].

Let $K$ be a weak* compact convex subset of the dual of a Banach space $X$. A subset $B$ of $K$ is called a boundary of $K$ if, for every $x \in X$, there exists an $x^{*} \in B$ such that $x^{*}(x)=\sup \left\{y^{*}(x): y^{*} \in K\right\}$. We shall say that $B(I)$-generates $K$ if, for every countable cover $\left\{C_{n}: n \in \mathbb{N}\right\}$ of $B$ by weak* compact convex subsets of $K$, the convex

(C) 2011 Australian Mathematical Publishing Association Inc. 0004-9727/2011 \$16.00 
hull of $\bigcup_{n \in \mathbb{N}} C_{n}$ is norm dense in $K$. Finally, we shall denote by $B_{X^{*}}$ the closed unit ball in $X^{*}$ and by $\operatorname{Ext}(K)$ the set of all extreme points of $K$.

The main theorem relies upon the following prerequisite result.

LEMma 1. Suppose that $K, S$ and $\left\{K_{n}: n \in \mathbb{N}\right\}$ are weak ${ }^{*}$ compact subsets of the dual of a Banach space $X$. Suppose also that $S \cap K=\varnothing$ and $S \subseteq{\overline{\bigcup_{n \in \mathbb{N}} K_{n}}}^{*}$. If, for each weak* open neighbourhood $W$ of 0 , there exists an $N \in \mathbb{N}$ such that $K_{n} \subseteq K+W$ for all $n>N$, then $S \subseteq \bigcup_{1 \leq n \leq M} K_{n}$ for some $M \in \mathbb{N}$.

PROOF. Since $K \cap S=\varnothing$ there exists a weak* open neighbourhood $W$ of 0 such that $K+W \subseteq X^{*} \backslash S$. By making $W$ smaller, we may assume that $K+\bar{W}^{w^{*}} \subseteq X^{*} \backslash S$. From the hypotheses there exists an $M \in \mathbb{N}$ such that $\bigcup_{n>M} K_{n} \subseteq K+W$ and so

$$
\bigcup_{n>M} K_{n} w^{*} \subseteq K+\bar{W}^{w^{*}} \subseteq X^{*} \backslash S \text { since } K+\bar{W}^{w^{*}} \text { is weak }{ }^{*} \text { closed. }
$$

On the other hand,

$$
S \subseteq \coprod_{n \in \mathbb{N}} K_{n} w^{*}={\overline{\bigcup_{n>M} K_{n}}}^{w^{*}} \cup \bigcup_{1 \leq n \leq M} K_{n}
$$

Therefore, $S \subseteq \bigcup_{1 \leq n \leq M} K_{n}$.

We may now state and prove the main theorem.

THEOREM 2. Let $K$ be a weak ${ }^{*}$ compact convex subset of the dual of a Banach space $X$ and let $B$ be a boundary of $K$. Then $B(I)$-generates $K$.

PROOF. After possibly translating $K$ we may assume that $0 \in B$. Suppose that $B \subseteq \bigcup_{n \in \mathbb{N}} C_{n}$ where $\left\{C_{n}: n \in \mathbb{N}\right\}$ are weak* compact convex subsets of $K$. Fix $\varepsilon>0$. We will show that

$$
K \subseteq \operatorname{co}\left[\bigcup_{n \in \mathbb{N}} C_{n}\right]+2 \varepsilon B_{X^{*}}
$$

For each $n \in \mathbb{N}$, let $K_{n}:=C_{n}+(\varepsilon / n) B_{X^{*}}$ and let $V^{*}:=\overline{\mathrm{co}} w^{*} \bigcup_{n \in \mathbb{N}} K_{n}$. Clearly, $B \subseteq \bigcup_{n \in \mathbb{N}} K_{n}$ and so $K=\overline{\mathrm{co}} w^{*}(B) \subseteq V^{*}$. It is also clear that $V^{*}$ is a weak* compact convex body in $X^{*}$ with $0 \in \operatorname{int}\left(V^{*}\right)$. Let $x^{*}$ be any element of

$$
\Sigma_{V}:=\left\{y^{*} \in V^{*}: \widehat{x}\left(y^{*}\right)=\max _{z^{*} \in V^{*}} \widehat{x}\left(z^{*}\right) \text { for some } x \in X \backslash\{0\}\right\}
$$

and let $x \in X$ be chosen so that $\widehat{x}\left(x^{*}\right)=\max _{z^{*} \in V^{*}} \widehat{x}\left(z^{*}\right)=1$. It is easy to see that if $F:=\left\{y^{*} \in V^{*}: y^{*}(x)=1\right\}$ then $F \cap K=\varnothing$. Indeed, if $F \cap K \neq \varnothing$ then $\max \left\{y^{*}(x)\right.$ : $\left.y^{*} \in K\right\}=1$ and because $B$ is a boundary for $K$ it follows that, for some $j \in \mathbb{N}$, there is a $b^{*} \in C_{j} \cap B$ such that $b^{*}(x)=1$. However, as $b^{*} \in b^{*}+(\varepsilon / j) B_{X^{*}} \subseteq K_{j} \subseteq V^{*}$, 
this is impossible. Now,

$$
\begin{aligned}
\operatorname{Ext}(F) & \subseteq \operatorname{Ext}\left(V^{*}\right) \quad \text { since } F \text { is an extremal subset of } V^{*} \\
& \subseteq \bigcup_{n \in \mathbb{N}} K_{n} w^{*} \quad \text { by Milman's theorem. }
\end{aligned}
$$

Thus,

$$
\operatorname{Ext}(F) \subseteq F \cap{\overline{\bigcup_{n \in \mathbb{N}} K_{n}}}^{w^{*}} \subseteq \widehat{\bigcup}_{n \in \mathbb{N}} K_{n} w^{*}
$$

and so by Lemma 1 , applied to the weak* compact set $S:=F \cap{\overline{\bigcup_{n \in \mathbb{N}} K_{n}}}^{w^{*}}$, there exists an $M \in \mathbb{N}$ such that $\operatorname{Ext}(F) \subseteq S \subseteq \bigcup_{1 \leq n \leq M} K_{n}$. Hence,

$$
\begin{aligned}
x^{*} \in F=\overline{\mathrm{co}}^{w^{*}} \operatorname{Ext}(F) \subseteq \operatorname{co}\left[\bigcup_{1 \leq n \leq M} K_{n}\right] & \subseteq \operatorname{co}\left[\bigcup_{1 \leq n \leq M} C_{n}\right]+\varepsilon B_{X^{*}} \\
& \subseteq \operatorname{co}\left[\bigcup_{n \in \mathbb{N}} C_{n}\right]+\varepsilon B_{X^{*}} .
\end{aligned}
$$

Since $x^{*} \in \Sigma_{V}$ was arbitrary, we have by the Bishop-Phelps theorem, which says that $\Sigma_{V}$ is dense in $\partial V^{*}$, that

$$
\partial V^{*} \subseteq \operatorname{co}\left[\bigcup_{n \in \mathbb{N}} C_{n}\right]+2 \varepsilon B_{X^{*}}
$$

However, since $0 \in B$ (and hence in some $C_{n}$ ) it follows that $K \subseteq V^{*} \subseteq$ $\operatorname{co}\left[\bigcup_{n \in \mathbb{N}} C_{n}\right]+2 \varepsilon B_{X^{*}}$. Since $\varepsilon>0$ was arbitrary we are done.

There are many applications of this theorem. In particular, we have the following.

Corollary 3. Let $K$ be a weak ${ }^{*}$ compact convex subset of the dual of a Banach space $X$, let $B$ be a boundary for $K$ and let $f_{n}: K \rightarrow[0, \infty)$ be weak ${ }^{*}$ lower semicontinuous convex functions. If $\left\{f_{n}: n \in \mathbb{N}\right\}$ are equicontinuous with respect to the norm and $\lim _{n \rightarrow \infty} f_{n}\left(b^{*}\right)=0$ for each $b^{*} \in B$, then $\lim _{n \rightarrow \infty} f_{n}\left(x^{*}\right)=0$ for each $x^{*} \in K$.

PROOF. Clearly, it is sufficient to show that $\lim _{\sup } \rightarrow \infty f_{n}\left(x^{*}\right)=0$ for each $x^{*} \in K$. To this end, fix $\varepsilon>0$. For each $n \in \mathbb{N}$, let

$$
C_{n}:=\left\{y^{*} \in K: f_{k}\left(y^{*}\right) \leq \varepsilon / 2 \text { for all } k \geq n\right\} .
$$

Then $\left\{C_{n}: n \in \mathbb{N}\right\}$ is a countable cover of $B$ by weak* compact convex subsets of $K$. Therefore, $\operatorname{co}\left[\bigcup_{n \in \mathbb{N}} C_{n}\right]=\bigcup_{n \in \mathbb{N}} C_{n}$ is norm dense in $K$. Since $\left\{f_{n}: n \in \mathbb{N}\right\}$ are equicontinuous (with respect to the norm) it follows that $\lim _{\sup _{n \rightarrow \infty}} f_{n}\left(x^{*}\right)<\varepsilon$ for all $x^{*} \in K$. 
The classical Rainwater's theorem [13] follows from this by setting $K:=B_{X^{*}}$ and $B:=\operatorname{Ext}(K)$ and, for any bounded set $\left\{x_{n}: n \in \mathbb{N}\right\}$ in $X$ that converges to $x \in X$ with respect to the topology of pointwise convergence on $\operatorname{Ext}\left(B_{X^{*}}\right)$, letting $f_{n}: K \rightarrow$ $[0, \infty)$ be defined by $f_{n}\left(x^{*}\right):=\left|x^{*}\left(x_{n}\right)-x^{*}(x)\right|$.

As promised, we give a simple proof of James' theorem valid for separable, closed and bounded convex sets. In the proof of this theorem we shall denote the natural embedding of a Banach space $X$ into its second dual $X^{* *}$ by $\widehat{X}$, and similarly we shall denote the natural embedding of an element $x \in X$ by $\widehat{x}$.

THEOREM 4. Let $C$ be a closed and bounded convex subset of a Banach space $X$. If $C$ is separable and every continuous linear functional on $X$ attains its supremum over $C$ then $C$ is weakly compact.

Proof. Let $K:=\widehat{\widehat{C}}^{w^{*}}$. To show that $C$ is weakly compact it is sufficient to show that,

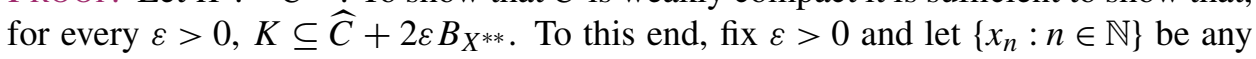
dense subset of $C$. For each $n \in \mathbb{N}$, let $C_{n}:=K \cap\left[\widehat{x_{n}}+\varepsilon B_{X^{* *}}\right]$. Then $\left\{C_{n}: n \in \mathbb{N}\right\}$ is a cover of $\widehat{C}$ by weak ${ }^{*}$ closed convex subsets of $K$. Since $\widehat{C}$ is a boundary of $K$, $K \subseteq \overline{\mathrm{co}} \bigcup_{n \in \mathbb{N}} C_{n} \subseteq \widehat{C}+2 \varepsilon B_{X^{* *}}$.

The notion of (I)-generation has been used by some other authors (see [7]) to provide a proof of the full version of James' theorem without recourse to Simons' inequality.

If we are willing to invest a little more effort we can extend Theorem 4 to the setting where $B_{X^{*}}$ is weak* sequentially compact. To see this we need the following lemma.

LEMMA 5. Let $C$ be a closed and bounded convex subset of a Banach space X. If $\left(B_{X^{*}}\right.$, weak*) is sequentially compact and every continuous linear functional on $X$ attains its supremum over $C$ then, for each $\mathcal{F} \in B_{X^{* * *}}$, there exists an $x^{*} \in B_{X^{*}}$ such that

$$
\left.\mathcal{F}\right|_{\widehat{C} w^{*}}=\left.\widehat{x^{*}}\right|_{\widehat{C}} w^{*}
$$

ProOf. Let $K:=\bar{C}^{w^{*}}$ and note that $\widehat{C}$ is a boundary of $K$. Let $B_{p}(K)\left(C_{p}(K)\right)$ denote the bounded real-valued (weak* continuous real-valued) functions defined on $K$, endowed with the topology of pointwise convergence on $K$. For an arbitrary subset $Y$ of $K$, let $\tau_{p}(Y)$ denote the topology on $B(K)$ of pointwise convergence on $Y$. Consider $S:\left(B_{X^{*}}\right.$, weak $\left.{ }^{*}\right) \rightarrow\left(C(K), \tau_{p}(\widehat{C})\right)$ defined by $S\left(x^{*}\right):=\left.\widehat{x^{*}}\right|_{K}$. Since $S$ is continuous, $S\left(B_{X^{*}}\right)$ is sequentially $\tau_{p}(\widehat{C})$-compact. Hence, from Corollary $3, S\left(B_{X^{*}}\right)$ is sequentially $\tau_{p}(K)$-compact. It then follows from Grothendieck's theorem [1, Ch. 4] that $S\left(B_{X^{*}}\right)$ is a compact subset of $C_{p}(K)$ and so a compact subset of $B_{p}(K)$. In particular, $S\left(B_{X^{*}}\right)$ is a closed subset of $B_{p}(K)$. Next, consider $T:\left(B_{X^{* * *}}\right.$, weak $\left.{ }^{*}\right) \rightarrow$ $B_{p}(K)$ defined by $T(\mathcal{F}):=\left.\mathcal{F}\right|_{K}$. Then $T$ is continuous and so $T\left(B_{\widehat{X}^{*}}\right)$ is dense in $T\left(B_{X^{* * *}}\right)$, since $B_{X^{*}}$ is weak ${ }^{*}$ dense in $B_{X^{* * *}}$ by Goldstine's theorem. However, $T\left(B_{X^{*}}\right)=S\left(B_{X^{*}}\right)$, which is closed in $B_{p}(K)$. Therefore, $T\left(B_{X^{* * *}}\right)=S\left(B_{X^{*}}\right)=$ $T\left(B_{X^{*}}\right)$. This completes the proof. 
THEOREM 6. Let $C$ be a closed and bounded convex subset of a Banach space $X$. If $\left(B_{X^{*}}\right.$, weak $\left.{ }^{*}\right)$ is sequentially compact and every continuous linear functional on $X$ attains its supremum over $C$ then $C$ is weakly compact.

PRoOF. Let $K:=\widehat{C}^{w^{*}}$. In order to obtain a contradiction, suppose that $\widehat{C} \subsetneq K$. Let $F \in K \backslash \widehat{C}$. Then there exists a $\mathcal{F} \in B_{X^{* * *}}$ such that $\mathcal{F}(F)>\sup _{\widehat{c} \in \widehat{C}} \widehat{\mathcal{F}}(\widehat{c})$. However, by Lemma 5 there exists an $x^{*} \in B_{X^{*}}$ such that $\left.\widehat{x^{*}}\right|_{K}=\left.\mathcal{F}\right|_{K}$. Therefore,

$$
\widehat{x^{*}}(F)=\mathcal{F}(F)>\sup _{\widehat{c} \in \widehat{C}} \mathcal{F}(\widehat{c})=\sup _{\widehat{c} \in \widehat{C}} \widehat{x^{*}}(\widehat{c})=\max _{G \in K} \widehat{x^{*}}(G),
$$

which contradicts the fact that $F \in K$. Therefore, $K=\widehat{C}$ and so $C$ is weakly compact.

For some related articles, see [10-12].

\section{References}

[1] A. V. Arkhangel'skii, Topological Function Space, Mathematics and its Applications (Soviet Series), 78 (Kluwer Academic Publishers Group, Dordrecht, 1992).

[2] E. Bishop and R. Phelps, The Support Functionals of a Convex Set, Proceedings of Symposia in Pure Mathematics, VII (American Mathematical Society, Providence, RI, 1963), pp. 27-35.

[3] V. Fonf and J. Lindenstrauss, 'Boundaries and generation of convex sets', Israel J. Math. 136 (2003), 157-172.

[4] V. Fonf, J. Lindenstrauss and R. Phelps, 'Infinite dimensional convexity', in: Handbook of the Geometry of Banach Spaces, Vol. I (North-Holland, Amsterdam, 2001), pp. 599-670.

[5] G. Godefroy, 'Boundaries of convex sets and interpolation sets', Math. Ann. 277 (1987), 173-184.

[6] R. C. James, 'Weakly compact sets', Trans. Amer. Math. Soc. 113 (1964), 129-140.

[7] O. Kalenda, ' $(I)$-envelopes of the unit balls and James' characterisation of reflexivity', Studia Math. 182 (2007), 29-40.

[8] M. Krein and D. Milman, 'On the extreme points of regular convex sets', Studia Math. 9 (1940), 133-138.

[9] D. Milman, 'Characteristics of extremal points or regularly convex sets', Dokl. Akad. Nauk SSSR (N.S.) 57 (1947), 119-122.

[10] W. B. Moors, 'A characterisation of weak compactness in Banach spaces', Bull. Aust. Math. Soc. 55 (1997), 497-501.

[11] W. B. Moors and E. A. Reznichenko, 'Separable subspaces of affine function spaces on convex compact set', Topology Appl. 155 (2008), 1306-1322.

[12] W. B. Moors and J. Spurný, 'On the topology of pointwise convergence on the boundaries of $L_{1}$-preduals', Proc. Amer. Math. Soc. 137 (2009), 1421-1429.

[13] J. Rainwater, 'Weak convergence of bounded sequences', Proc. Amer. Math. Soc. 14 (1963), 999.

[14] S. Simons, 'A convergence theorem with boundary', Pacific J. Math. 40 (1972), 703-708.

WARREN B. MOORS, Department of Mathematics, The University of Auckland, Private Bag 92019, Auckland, New Zealand

e-mail: moors@math.auckland.ac.nz 